\title{
Pallidal Stimulation in Parkinson's Disease Patients with a Prior Unilateral Pallidotomy
}

\author{
Néstor Gálvez-Jiménez, Andres Lozano, Ronald Tasker, Janice Duff, \\ William Hutchison and Anthony E. Lang
}

\begin{abstract}
Objective: To evaluate, in a double-blind fashion, the efficacy of deep brain stimulation of the internal segment of the globus pallidus (GPi) contralateral to a previous unilateral medial pallidotomy (MP). Methods: This pilot study involved 4 patients with a previous MP and one previously unoperated patient unable to tolerate any antiparkinsonian drugs. One of the patients with a prior unilateral MP had two electrodes implanted in the contralateral side, one in GPi and one in Vim thalamus. Detailed neurologic assessments were performed after overnight drug withdrawal and in the drug "on" state at baseline, 1 week and 3 months (in all), and 9 and 12 months (one) with patients and evaluators blinded to the status of stimulation. Results: GPi stimulation resulted in improvements in "off-period" contralateral bradykinesia, rigidity and tremor in all patients. Dyskinesias and freezing episodes were ameliorated in one patient each but dyskinesias were transiently induced in another. The patient with GPi+Vim electrodes had complete resolution of contralateral tremor with thalamic stimulation but less benefit from acute GPi stimulation. One patient experienced a single seizure one week post-op and no other surgical complications were observed. Conclusions: Deep brain stimulation can be applied safely and effectively in patients who have already had a pallidotomy on the contralateral side. The effect of stimulation at different sites on different symptom profiles and levodopa-induced dyskinesias requires further evaluation.
\end{abstract}

RÉSUMÉ: Stimulation pallidale chez les parkinsoniens ayant subi une pallidotomie unilatérale antérieurement. But: D'évaluer, par une étude à double insu, l'efficacité d'une stimulation du segment interne du globus pallidus (GPi) contralatéral à une pallidotomie médiale (PM) unilatérale subie antérieurement. Méthodes: Cette étude pilote porte sur 4 patients ayant subi une PM antérieurement et un patient qui n'avait pas subi de chirurgie et qui ne tolérait aucun médicament antiparkinsonien. Chez un des patients qui avait subi une PM unilatérale, on a implanté deux électrodes du côté opposé, une dans le GPi et une dans le thalamus Vim. On a procédé à une évaluation neurologique détaillée alors que les patients n'avaient pas reçu leur médication depuis la veille et sous médication, en période "on", à 1 semaine, 3 mois (chez tous), 9 et 12 mois (chez un), la stimulation étant faite à l'insu des patients et des évaluateurs. Résultats: La stimulation GPi a provoqué une amélioration dans les périodes "off" de la bradykinésie, de la rigidité et du tremblement contralatéral chez tous les patients. Les dyskinésies ont été améliorées chez un patient et les épisodes de blocage chez un autre, mais des dyskinésies ont été induites de façon transitoire chez un autre. Le patient chez qui on avait implanté des électrodes GPi + Vim a eu une résolution complète du tremblement contralatéral par la stimulation thalamique, mais moins de bénéfice de la stimulation GPi aiguë. Un patient a eu une crise épileptique unique une semaine après l'intervention. Aucune autre complication chirurgicale n'a été observée. Conclusions: La stimulation de régions profondes du cerveau peut être faite de façon sécuritaire et efficace chez les patients qui ont déjà eu une pallidotomie du côté opposé. L'effet de la stimulation de différents sites sur différents profils de symptômes et sur les dyskinésies induites par la lévodopa demande une investigation plus poussée.

Can. J. Neurol. Sci. 1998; 25: 300-305

Pallidotomy has been found to be useful in the management of late-stage Parkinson's disease patients especially in those affected by severe disabling drug-induced dyskinesias. ${ }^{1-10}$ Improvement is obtained in symptoms such as off-period bradykinesia and rigidity with marked amelioration of druginduced dyskinesias. Recently, deep brain stimulation (DBS) has been proposed as an alternative to ablative lesioning for the management of many Parkinsonian symptoms and other movements disorders. ${ }^{11 \cdot 18}$ In preliminary studies, the delivery of high frequency deep brain stimulation (DBS) via bilaterally implanted electrodes in the globus pallidus internus $(\mathrm{GPi})^{19.21}$ or the

The Morton and Gloria Shulman Movement Disorders Centre, Division of Neurology and Playfair Neurosciences Unit, (N.G-J., J.D., A.E.L.); Division of Neurosurgery, (A.L., R.T.); Department of Physiology, (W.H.) The Toronto Hospital and The University of Toronto, Toronto, Ontario, Canada. RECEIVED FEBRUARY 24, 1998. ACCEPTED IN FINAL FORM JUNE 15, 1998.

Reprint requests to: Anthony E. Lang, The Morton and Gloria Shulman Movement Disorders Centre, The Toronto Hospital, 399 Bathurst Street, MP11-306, Toronto, Ontario, Canada M5T 2S8 
STN ${ }^{16}$ has been shown to improve symptoms of Parkinsonism in humans and in the MPTP treated monkey, ${ }^{22,23}$ although not all reports have been favourable. ${ }^{24}$ The advantage of DBS is the reversible and adaptable nature of the stimulation where different variables of the stimulus can be adjusted as required. Another potential benefit is that there is a negligible lesion produced during electrode implantation compared with the standard destructive neurosurgical procedure possibly resulting in fewer permanent complications.

Patients who have had a unilateral pallidotomy may experience increasing disability due to persistent and progressive signs on the unoperated side. Many neurosurgeons are reluctant to perform bilateral destructive lesions based on experience with bilateral thalamotomies where major morbidity can occur, particularly cognitive, memory and language, and swallowing and speech disturbances after a second stage bilateral procedure. ${ }^{25}$ Our initial experience in a small number of patients undergoing bilateral pallidotomy has confirmed the potential for immediate and long-term neuropsychological effects. ${ }^{26}$ Indeed, there are very few reliable data in the recent literature supporting the safety or efficacy of bilateral medial pallidotomy. ${ }^{27}$

We reasoned that patients with a prior unilateral pallidotomy who required another lesioning procedure could benefit from a less "destructive" approach. The "reversible" nature of stimulation permits the physician to tailor the treatment according to patient symptoms and response. If an undesirable side effect is observed during stimulation it can be easily corrected by adjusting the stimulation parameters. Therefore, implanting contralateral DBS electrodes in patients with a prior pallidotomy is a reasonable alternative to a bilateral lesioning procedure.

We present our preliminary short-term experience using DBS in 4 patients who had a prior unilateral pallidotomy. A second procedure contralateral to the initial pallidotomy was deemed necessary for these patients because of persistent disabling ipsilateral parkinsonian symptomatology and drug-induced dyskinesias. A fifth patient, who could not tolerate dopaminergic drugs, had a quadripolar electrode implanted into the GPi as the primary management of his parkinsonism.

\section{Patients And Methods}

Four men and a woman, with a mean age of 63 years and mean duration of Parkinson's disease of 10.4 years were included in the study (Table 1). Four patients had undergone a prior left pallidotomy on average 15 months earlier (range: 10 to 24 months) with improvement experienced predominantly on the side contralateral to the surgery. All continued to have severe and medically refractory parkinsonian symptoms on the unoperated ipsilateral side. All had asked that a second procedure be performed. A fifth patient with severe intolerance to antiparkinson medication underwent unilateral GPi implantation as the primary therapeutic modality for his disabling symptoms. Patients gave written informed consent and the study was approved by The Toronto Hospital Committee for Research on Human Subjects.

All patients had their antiparkinson drugs withheld the night before surgery. The surgical methods used were as reported previously by our group ${ }^{4,28,29}$ using stereotactic MRI in combination with microelectrode mapping of the target areas. These included the GPi in all patients and the Vim thalamus in one patient. This latter patient (\#5 on Table 1) had disabling rest tremor as well as rigidity and bradykinesia. In the final step, instead of performing a lesioning procedure, a permanent four channel DBS electrode (Medtronic model 3382) was inserted into the GPi in all patients (Figure 1) and a second electrode was inserted in the ipsilateral Vim in patient \#5. The electrode cable(s) was exteriorized for one week to allow immediate postoperative DBS testing. The cables were then internalized and connected to either an external (Medtronic Xtrel model 3425) or internal (Medtronic Itrel model 7424) stimulator.

Patients were assessed using the Unified Parkinson's Disease Rating Scale (UPDRS) ${ }^{30}$ both with and without stimulation. Stimulation parameters adjusted in each patient included electrode combinations (bipolar or unipolar), frequency, pulse width and amplitude of stimulation. Evaluations of stimulation effects in the practically defined worse off state after an overnight withdrawal of antiparkinson medication were performed in a random order with both rater and patient blinded to whether stimulator was ON or OFF. In order to maintain consistency, the same rater (JD) evaluated all patients throughout the study. Prior to these blinded evaluations the best stimulator $O N$ state was determined when both the patient and investigators agreed that the state represented the maximal therapeutic benefit as demonstrated by the total score of the motor subsection of the UPDRS. Specific features of the UPDRS chosen for reporting included the composite score for activities of daily living, and the motor subsections for tremor (items 20-21), rigidity (item 22), bradykinesia (items 23 26), postural stability plus gait disturbances (items 29-30) and the total motor score (Items 18-31). Dyskinesias were evaluated using a modified Obeso scale. ${ }^{31}$ (The status of drug treatment will be referred to using the lower case "off" and "on" while the stimulator status will be referred to using the upper case OFF

Table 1: Patient Characteristics.

\begin{tabular}{|c|c|c|c|c|c|c|c|c|}
\hline $\begin{array}{l}\text { PATIENT } \\
\text { NUMBER }\end{array}$ & SEX & AGE & $\begin{array}{l}\text { DISEASE } \\
\text { DURATION }\end{array}$ & $\begin{array}{l}\text { PREVIOUS } \\
\text { PALLIDOTOMY }\end{array}$ & $\begin{array}{l}\text { PREDOMINANT } \\
\text { SYMPTOMS* }\end{array}$ & $\begin{array}{l}\text { ELECTRODE } \\
\text { SITES }\end{array}$ & $\begin{array}{l}\text { FINAL } \\
\text { STIMULATION } \\
\text { SITE }\end{array}$ & $\begin{array}{l}\text { FOLLOW/UP } \\
\text { TIMES }\end{array}$ \\
\hline 2 & $\mathbf{M}$ & $51 y$ & $13 y$ & Left & $B / R / G$ & GPi & GPi & $3,9,12 \mathrm{M}$ \\
\hline 3 & $\mathbf{M}$ & $59 \mathrm{y}$ & $10 y$ & Left & $\mathrm{B} / \mathrm{R} / \mathrm{G} / \mathrm{Dys}$ & $\mathrm{GPi}$ & GPi & $3 \mathrm{M}$ \\
\hline 4 & $\mathbf{M}$ & $65 y$ & $4 y$ & NIL & $\mathrm{B} / \mathrm{R} / \mathrm{T}$ & GPi & $\mathrm{GPi}$ & $3 \mathrm{M}$ \\
\hline
\end{tabular}

${ }^{*} \mathrm{~B}=$ Bradykinesia, $\mathrm{R}=$ rigidity, $\mathrm{G}=$ gait, $\mathrm{T}=$ tremor, $\mathrm{Dys}=$ dyskinesias, $\mathrm{GPi}=$ globus pallidus, Vim = Ventral intermediate nucleus of the thalamus 


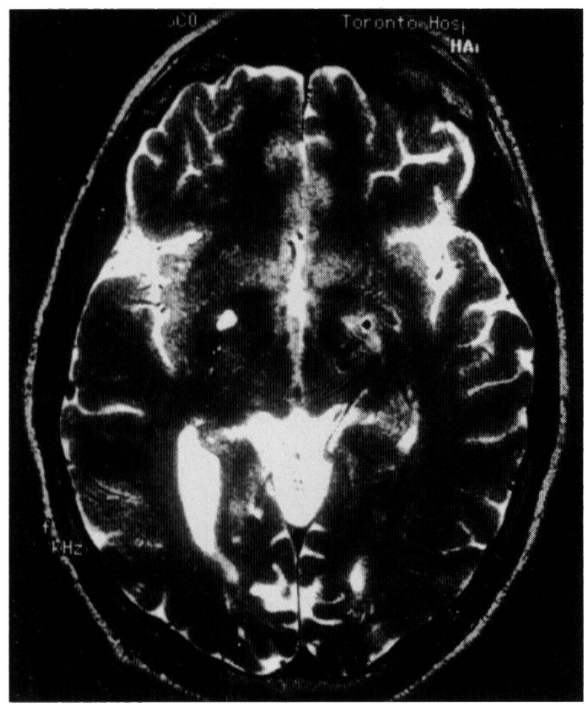

Figure: T2 weighted MRI (Patient 2) 5 days after implantation of the $D B S$ electrode showing the previous right pallidotomy (high intensity lesion) and the location of deep brain stimulation electrode in the GPi on the left.

and $\mathrm{ON}$.) In order to avoid the confounding effects of a persistent micropallidotomy causing improvement in symptoms postmicrorecording and electrode implantation, only scores obtained OFF and ON stimulation at the follow-up time points were compared (i.e., rather than comparing the post-op ON stimulation scores to baseline scores).

Follow up assessments were performed in all patients at one week after implantation of electrodes for chronic GPi stimulation. Four patients completed 3 months follow up and one patient completed 9 and 12 months. In all patients antiparkinson medication remained unchanged over the follow-up period.

The predominant symptoms and treatment characteristics for each patient are listed in Table 1. Bradykinesia and rigidity were present in all patients, followed by major gait disturbances in three patients and tremor and drug-induced dyskinesias in two patients. The GPi was the final site of chronic stimulation in four patients. One patient (\#5), who had both thalamic and GPi electrode implantation, chose Vim thalamus (after careful blinded assessments of both sites) as the permanent site for stimulation due to the greater beneficial effects obtained on her disabling contralateral tremor compared to the results observed in her rigidity and bradykinesia.

\section{Results}

\section{GPi stimulation}

There were no obvious changes seen in UPDRS scores with stimulation when the patients were in their "best on" state, having taken their usual doses of antiparkinsonian medications. Changes in dyskinesias in two patients are described below. Unless otherwise stated, the scores reported are for the practically defined "worse off" state with the patient off all antiparkinson medications overnight for a minimum of twelve hours. The total motor scores are provided in Table 2 . Four patients completed a 3 month follow up of chronic GPi stimulation, and one of these (patient 2) completed 9 months and 12 months. GPi stimulation resulted in a mean of $33 \%$ improvement in the total motor scores at one week compared to the stimulator in the OFF state in all five patients and $33 \%$ improvement at 3 months in 4 patients. In patient 2 there was a $25 \%$ improvement at 12 months.

Subscores of the motor component of the UPDRS are presented in Table 2. Evaluation of these was complicated by a variety of factors. For example, the difference between the preoperative total motor UPDRS scores and the 1 week stimulation OFF scores, as well as the increase in total motor OFF scores between one week and three months (Table 2) suggest the occurrence and subsequent decline of a beneficial micropallidotomy effect from the mapping and implantation surgery. Further evidence for a partial lesion effect was seen in patient 1 where contralateral limb rigidity, which was present at baseline, was barely evident at 1 week and absent at 3 months post-op and in

Table 2: UPDRS Scores*

\begin{tabular}{|c|c|c|c|c|c|c|c|c|c|c|c|}
\hline $\begin{array}{l}\text { Patient } \\
\text { Number }\end{array}$ & $\begin{array}{l}\text { PRE- } \\
\text { OP }\end{array}$ & $\begin{array}{l}1 \mathrm{WK} \\
\text { OFF/ON }\end{array}$ & $\begin{array}{l}3 \mathrm{M} \\
\text { OFF/ON }\end{array}$ & $\begin{array}{l}1 \mathrm{WK} \\
\text { OFF/ON }\end{array}$ & $\begin{array}{l}3 \mathrm{M} \\
\text { OFF/ON }\end{array}$ & $\begin{array}{l}1 \mathrm{WK} \\
\text { OFF/ON }\end{array}$ & $\begin{array}{l}3 \mathrm{M} \\
\text { OFF/ON }\end{array}$ & $\begin{array}{l}1 \mathrm{WK} \\
\text { OFF/ON }\end{array}$ & $\begin{array}{l}3 \mathrm{M} \\
\text { OFF/ON }\end{array}$ & $\begin{array}{l}1 \mathrm{WK} \\
\text { OFF/ON }\end{array}$ & $\begin{array}{l}3 \mathrm{M} \\
\text { OFF/ON }\end{array}$ \\
\hline 2 & 75 & $\begin{array}{l}38.0 / 20.0 \\
9 \mathrm{M} \\
38.5 / 35.0\end{array}$ & $\begin{array}{l}44.0 / 38.0 \\
12 \mathrm{M} \\
33.0 / 24.5\end{array}$ & $\begin{array}{l}3.0 / 1.0 \\
9 \mathrm{M} \\
2.0 / 2.0\end{array}$ & $\begin{array}{l}2.0 / 2.0 \\
12 \mathrm{M} \\
2.0 / 1.5\end{array}$ & $\begin{array}{l}11.0 / 6.0 \\
9 \mathrm{M} \\
8.5 / 7.0\end{array}$ & $\begin{array}{l}9.0 / 7.5 \\
12 \mathrm{M} \\
6.0 / 6.5\end{array}$ & $\begin{array}{l}2.0 / 0.0 \\
9 \mathrm{M} \\
0.0 / 0.0\end{array}$ & $\begin{array}{l}1.0 / 0.0 \\
12 \mathrm{M} \\
0.0 / 0.0\end{array}$ & $\begin{array}{l}3.5 / 0.0 \\
9 \mathrm{M} \\
2.0 / 1.0\end{array}$ & $\begin{array}{l}4.5 / 2.0 \\
12 \mathrm{M} \\
1.0 / 0.0\end{array}$ \\
\hline 3 & 51 & $18.5 / 17.0$ & $43.0 / 34.0$ & $2.0 / 2.0$ & $4.0 / 2.5$ & $3.5 / 3.0$ & $10.5 / 6.5$ & $1.0 / 0.0$ & $0.0 / 0.0$ & $2.5 / 1.5$ & $4.5 / 3.5$ \\
\hline 4 & 31.5 & $15.0 / 7.5$ & $29.0 / 9.0$ & $1.0 / 0.0$ & $2.0 / 0.0$ & $8.5 / 2.5$ & $11.5 / 6.0$ & $4.0 / 2.0$ & $8.0 / 0.0$ & $2.5 / 0.0$ & $1.5 / 0.0$ \\
\hline 5 & 37 & $41.0 / 25.0$ & $\mathrm{~N} / \mathrm{A}$ & $2.0 / 1.0$ & N/A & $7.0 / 3.0$ & $\mathrm{~N} / \mathrm{A}$ & $4.0 / 1.0$ & N/A & $4.0 / 4.0$ & N/A \\
\hline $\begin{array}{l}\text { MEANS } \\
(+/-S D)\end{array}$ & $\begin{array}{l}50.5 \\
(17.3)\end{array}$ & $\begin{array}{l}27.6(11.6) / \\
18.4(6.78)\end{array}$ & $\begin{array}{l}39.1(6.91) / \\
26.1(13.0)\end{array}$ & $\begin{array}{l}1.8(0.83) / \\
0.8(0.83)\end{array}$ & $\begin{array}{l}2.0(1.63) / \\
1.13(1.31)\end{array}$ & $\begin{array}{l}7.0(2.94) / \\
3.4(1.47)\end{array}$ & $\begin{array}{l}9.3(2.17) / \\
5.8(1.70)\end{array}$ & $\begin{array}{c}2.4(1.52) / \\
0.6(0.89)\end{array}$ & $\begin{array}{l}3.5(3.7) / \\
0.25(.50)\end{array}$ & $\begin{array}{l}3.4(0.89) / \\
1.8(1.89)\end{array}$ & $\begin{array}{r}3.8(1.50) / \\
2.3(1.66)\end{array}$ \\
\hline
\end{tabular}

*Scores obtained in drug-off state (patient withdrawn overnight for at least 12 hours), evaluated by rater blinded to whether stimulator was ON or OFF. Rigidity, Bradykinesia and Tremor Scores are for the limbs contralateral to the GPi stimulator.

**Postural stability 
patient 3 tremor was minimal at 1 week and absent at the 3 months follow-up. Patient 2, followed for 12 months, demonstrated a declining effect of stimulation on the composite bradykinesia scores over that time (comparing the stimulator OFF to ON scores). However, stimulation OFF bradykinesia scores had slowly declined and the 12 months score was identical to the stimulation ON score at week 1 (which was $60 \%$ less than the stimulation OFF score at that time point). This change suggests a degree of cumulative or "carryover" effect on bradykinesia of chronic pallidal stimulation.

Contralateral tremor improved in response to GPi stimulation by an average of $75 \%$ at 1 week in all five patients and $93 \%$ at 3 months in the 3 patients demonstrating tremor at that time point. The contralateral "off" state bradykinesia composite score improved an average of $51 \%$ with the stimulator ON at 1 week and $37 \%$ at 3 months. Gait and postural stability composite scores showed an overall improvement at one week of $47 \%$ and $40 \%$ at 3 months. Patient 2 showed the most striking changes with sustained benefit for up to 12 months. There was a $50 \%$ improvement at 9 months and complete amelioration of gait disturbances at 12 months. Again, changes in stimulation OFF scores suggested a carryover effect of prolonged pallidal stimulation.

Activities of daily living (ADL) scores were not routinely obtained at the times of blinded motor evaluations. These scores were available at variable follow-up time points. At 1 week GPi stimulation in the 4 patients subsequently undergoing chronic stimulation at this site resulted in a $76 \%$ improvement in offperiod ADL scores (mean +/- standard deviation: STIM OFF = $22+/-6.28$, STIM ON $=5.25+/-6.25$ ). ADL scores were available at 3 months for 2 of these 4 patients and at 6 months and 12 months in one-each. There was an average improvement of $65 \%$ combining these time points (STIM OFF $=22.5+/-6.52$; STIM $\mathrm{ON}=7.87+/ .6 .81$ ).

The response of drug-induced dyskinesias to GPi stimulation in patients 1 and 3 was evaluated in the drug-on state. Contralateral dyskinesias were consistently ameliorated in patient 1 . At 1 week there was complete resolution of dyskinesias with the stimulator ON (contralateral dyskinesia score (maximum $=4$ ) STIM OFF $=2$; STIM ON $=0$ ); and at 3 months they improved by $50 \%$ (STIM OFF $=2$; STIM ON $=1$ ). In patient 3 there was consistent but transient worsening of drug-induced dyskinesias with the stimulator ON as seen at 1 week (STIM $\mathrm{OFF}=3$; STIM ON = 4). However, it was possible to reprogram the stimulator to improve his parkinsonian symptoms at a lower stimulation voltage maintaining the antiparkinsonian effect without a pronounced increase in dyskinesias. Furthermore, in this patient mild contralateral dyskinesias were consistently induced by GPi stimulation in the practically defined worst-off state, even though the patient had taken no antiparkinsonian medications for over 12 hours (drugs off, STIM OFF $=0$; drugs off, STIM ON = 2).

We did not discern that varying the specific electrodes stimulated had differing effects on inducing or relieving dyskinesias.

\section{Thalamic stimulation (Patient 5).}

Patient 5 had simultaneous unilateral GPi and Vim electrodes implanted with testing of both in the first post-operative week. Acute GPi stimulation incompletely reduced scores for tremor, bradykinesia, and rigidity but not gait or postural stability (Table
2). Vim stimulation completely ameliorated the contralateral tremor but had little effect on other Parkinsonian scores. Based on this differential response, she chose to have chronic thalamic stimulation because tremor was her most disabling symptom. The GPi electrode was left in place and the connecting wires were buried under the skin. We have followed this patient for approximately 2 years since Vim implantation. Although tremor continues to respond well, other Parkinson symptoms are causing greater disability and we are now considering connecting the GPi electrode to a chronic stimulator.

\section{Complications}

Patient 2 (followed for 12 months) had a single seizure one week after surgery and was treated with routine anticonvulsant therapy for three months without recurrence. All patients experienced transient paresthesias at the highest levels of stimulation and one patient had a transient dose-limiting worsening of dysarthria during GPi stimulation. Patient 2 noted an initial flash of light when turning the GPi stimulator ON. No overt cognitive changes were noted after insertion of the electrodes or when stimulation was turned $\mathrm{ON}$.

\section{Discussion}

This pilot study involved a small number of patients with a relatively short follow-up. Despite this, we can draw some important tentative conclusions, particularly given the blinded evaluations of clinical efficacy performed. Our preliminary observations demonstrate that GPi stimulation can improve the major symptoms of PD and that DBS of the contralateral GPi or Vim thalamus in patients who have already had a destructive pallidotomy can be performed safely and successfully. We have also demonstrated in one patient the feasibility of implanting 2 quadripolar electrodes into 2 different sites on the same side. This allows post-operative testing in order to determine the optimum site(s) for subsequent stimulation.

This is the first study to report blinded evaluations of GPi deep brain stimulation and the first to report the use of DBS in patients who have had a previous unilateral pallidotomy. Although the improvement in scores between baseline and stim OFF at 1 week and the decline between the 1 week and 3 month stim OFF scores both suggest the occurrence of a waning micropallidotomy effect, this cannot account for the clear differences found between the stim ON and OFF states. One potentially important observation which may confound the future long-term evaluations of DBS in nonthalamic sites is the apparent cumulative or carryover effect seen in the patient with the longest follow-up. It is quite possible that long-term continuous DBS will induce adaptive changes in the basal ganglia which need to be considered when attempting to evaluate ongoing response to this therapy.

It is currently accepted that many of the symptoms of Parkinson's disease are due to the overactivity of the inhibitory pallidofugal pathways originating from the GPi; the GPi being driven in part by an overactive STN. The net effect is the inhibition of cortically-mediated impulses via the thalamocortical loop. ${ }^{32,-36}$ This is supported by recent positron emission tomography studies which have shown activation of nonprimary cortical motor areas (supplementary motor area, dorsolateral prefrontal cortex) in response to GPi and STN stimulation. ${ }^{37,38}$ Because of our current knowledge of their anatomical relationships and physiological 
functions and the ease with which these structures can be accessed through current neurosurgical techniques, the GPi, STN and thalamus are suitable targets in PD patients for electrode implantation and neuromodulation using DBS. In our patients, GPi stimulation regularly resulted in improvement in all major off-period symptoms on the side contralateral to the stimulation. GPi stimulation may also improve off-period axial symptoms such as gait and postural stability as seen in patient 2 . Furthermore, those parkinsonian patients who cannot tolerate medication despite best therapeutic efforts may benefit from DBS as primary treatment, as demonstrated by patient 4 .

We found the effects of GPi stimulation on dyskinesias to be complex and varied. Since this study was completed, Bejjani et al. ${ }^{39}$ and Krack et al ${ }^{40}$ have reported a differential response to stimulation in different parts of the pallidum: stimulation of the lower contact of the quadripolar electrode (in the posteroventral GP) dramatically reduced levodopa-induced dyskinesias but could worsen parkinsonism with the exception of rigidity and even block the beneficial effects of levodopa, while stimulation of the upper contact (in the dorsal GP, probably including the GPe) improved parkinsonism but could induce dyskinesias in the off state and worsen them in the on state. In our 2 patients, we had not observed a site-dependent response of dyskinesias although this question was not methodically tested as it was by Bejjani and Krack and their colleagues. As with pallidotomy, where lesion location and size may determine clinical response, it is reasonable to expect that the location of electrodes, parameters of stimulation (voltage, frequency and pulse width) and volume of tissue influenced by DBS may result in varying responses of parkinsonism and dyskinesias. Further studies in larger numbers of patients will be required to address these issues.

Experience in patient 5 provides preliminary evidence for the power of the double electrode implantation approach which allowed testing and comparisons of stimulation in two sites before committing to long-term therapy. Although we have obtained pronounced improvement in tremor with GPi stimulation, her response to Vim stimulation was greater. The retention of the non-stimulated electrode in situ (in this case in the GPi) provides the option of using this electrode for DBS at a later time when other symptoms begin to cause a greater proportion of the total disability. However, stimulation of the STN can result in pronounced benefit to all of the features of Parkinson's disease. ${ }^{16}$ Preliminary evidence suggests that STN stimulation may be as effective for tremor as stimulation of the Vim..$^{41}$ If, as our own recent personal experience suggests, this effect is consistently confirmed, then the STN may become the target of first choice for patients such as our case \#5, eliminating the advantage of being able to compare stimulation of Vim to an alternative site provided by double electrode implantation.

Our recent experience in two patients who had staged bilateral destructive pallidotomies, which improved their disabling drug-induced dyskinesias, but resulted in problematic cognitive disturbances ${ }^{26}$ supports the interaction of the basal ganglia with the orbitofrontal, limbic and dorsolateral prefrontal cortical areas. Thus, the performance of bilateral destructive lesioning procedures should only be performed after careful consideration of the potential risks involved, when the patient and relatives are aware of the possible serious, persistent adverse effects and only in those patients with symptoms of late-stage Parkinson's disease known to be responsive to the lesion in whom no other medical or surgical alternatives exist. DBS may provide a safer alternative to bilateral lesioning procedures for these patients.

Based on this pilot study the following preliminary conclusions can be made: a) implanting quadripolar electrodes into the contralateral GPi in patients who already had a unilateral pallidotomy is technically feasible and safe and may provide an alternative to bilateral ablative stereotactic procedures; b) the preliminary observations in patient 5 with implantation of both GPi and Vim quadripolar electrodes on the same side of the brain suggest that the double electrode implantation approach may be useful for determining the best site for subsequent chronic stimulation, although STN stimulation may become a better first choice in this situation; c) DBS contralateral to prior pallidotomy improves off period symptoms on the contralateral side and may also improve gait and postural stability; and d) GPi stimulation may be used as primary treatment of parkinsonian symptoms in patients unable to tolerate antiparkinsonian medications, as demonstrated in one of our patients. As the differential responses of specific symptoms of parkinsonism and levodopa-induced dyskinesias to stimulation at different anatomical sites are better elucidated, it should become possible to tailor the stimulation more effectively to patient needs. The response of dyskinesias ${ }^{39,40}$ and the neuropsychological effects ${ }^{42}$ of DBS need further exploration. In the future, rigorous prospective evaluation in a larger number of patients is required to determine duration of response and long-term effects.

\section{ACKNOWLEDGMENTS}

This work was supported by Medtronic, Minneapolis, MN, and the National Parkinson's Foundation, Miami, Fl and the Parkinson Foundation of Canada. Dr.Gálvez-Jiménez is the Ronald and Patty Rosenfeld traveling fellow of The Cleveland Clinic Foundation. Dr. A. Lozano is a Medical Research Council of Canada Clinician Scientist. The authors acknowledge the secretarial assistance of Shelley Malton in the preparation of this manuscript. None of the authors have a financial interest in Medtronic Neurological.

\section{REFERENCES}

1. Baron MS, Vitek JL, Bakay RAE, et al. Treatment of advanced Parkinson's disease by posterior GPi pallidotomy: 1-year results of a pilot study. Ann.Neurol. 1996; 40: 355-366.

2. Dogali M, Fazzini E, Kolodny E, et al. Stereotactic ventral pallidotomy for Parkinson's disease. Neurology 1995; 45: 753-761.

3. Laitinen LV, Bergenheim AT, Hariz MI. Leksell's posteroventral pallidotomy in the treatment of Parkinson's disease. J.Neurosurg. 1992; 76: 53-61.

4. Lang AE, Lozano AM, Montgomery E, et al. Posteroventral medial pallidotomy in advanced Parkinson's disease. N Engl J Med 1997; 337: 1036-1042.

5. Favre J, Taha JM, Nguyen TT, Gildenberg PL, Burchiel KJ. Pallidotomy: a survey of current practice in North America. Neurosurgery $1996 ; 39: 883-892$.

6. Fazzini E, Dogali M, Sterio D, Eidelberg D, Beric A. Stereotactic pallidotomy for Parkinson's disease: A long-term follow-up of unilateral pallidotomy. Neurology 1997; 48: 1273-1277.

7. Johansson F, Malm J, Nordh E, Hariz M. Usefulness of pallidotomy in advanced Parkinson's disease. J Neurol Neurosurg Psychiatry 1997; 62: 125-132.

8. Iacono RP, Lonser RR, Ulloth JE, Shima F. Postero-ventral pallidotomy in Parkinson's disease. J Clin Neurosci 1995; 2: 140-145.

9. Kishore A, Turnbull IM, Snow BJ, et al. Efficacy, stability and predictors of outcome of pallidotomy for Parkinson's disease - Sixmonth follow-up with additional 1-year observations. Brain 1997; 120: 729-737. 
10. Uitti RJ, Wharen RE, Turk MF, et al. Unilateral pallidotomy for Parkinson's disease: comparison of outcome in younger versus elderly patients. Neurology 1997; 49: 1072-1077.

11. Benabid AL, Pollack P, Gervason C, et al. Long-term suppression of tremor by chronic stimulation of the ventral intermediate thalamic nucleus. Lancet 1991; 337: 403-406.

12. Broggi G, Brock S, Franzini A, Geminiani G. A case of posttraumatic tremor treated by chronic stimulation of the thalamus. Mov Disord 1993; 8: 206-208.

13. Burleigh AL, Horak FB, Burchiel KJ, Nutt JG. Effects of thalamic stimulation on tremor, balance, and step initiation: a single subject study. Mov Disord 1993; 4: 519-524.

14. Caparros-Lefebvre D, Blond S, Vermersch $P$, et al. Chronic thalamic stimulation improves tremor and levodopa induced dyskinesias in Parkinson's disease. J Neurol Neurosurg Psychiatry 1993; 56: $268-273$.

15. Caparros-Lesebvre D, Ruchoux MM, Blond S, Petit H, Percheron G. Long-term thalamic stimulation in Parkinson's disease: postmortem anatomoclinical study. Neurology 1994; 44: 18561860.

16. Limousin P, Pollak P, Benazzouz A, et al. Effect on parkinsonian signs and symptoms of bilateral subthalamic nucleus stimulation. Lancet 1995; 345: 91-95.

17. Sellal F, Hirsch E, Barth $P$, Blond S, Marescaux C. A case of symptomatic hemidystonia improved by ventroposterolateral thalamic electrostimulation. Mov Disord 1993; 8: 515-518.

18. Tsubokawa T, Katayama Y, Yamamoto T. Control of persistent hemiballismus by chronic thalamic stimulation. Report of two cases. J Neurosurg 1995; 82: 501-505.

19. Pahwa R, Wilkinson S, Smith D, et al. High-frequency stimulation of the globus pallidus for the treatment of Parkinson's disease. Neurology 1997; 49: 249-253.

20. Siegfried J, Lippitz B. Bilateral chronic electrostimulation of ventroposterolateral pallidum: a new therapeutic approach for alleviating all parkinsonian symptoms. Neurosurgery 1994; 35 : $1126-1130$.

21. Gross C, Rougier A, Guehl D, et al. High-frequency stimulation of the globus pallidus internalis in Parkinson's disease: a study of seven cases. J Neurosurg 1997; 87: 491-498.

22. Benazzouz A, Gross C, Feger J, Bonard T, Bioulac B. Reversal of rigidity and improvement in motor performance by subthalamic high frequency stimulation in MPTP treated monkeys. Eur J Neurosci. 1993; 5: 383-389.

23. Boraud T, Bezard E, Bioulac B, Gross C. High frequency stimulation of the internal Globus Pallidus (GPi) simultaneously improves parkinsonian symptoms and reduces the firing frequency of GPi neurons in the MPTP-treated monkey. Neuroscience Letters 1996; 215: 17-20.

24. Tronnier VM, Fogel W, Kronenbuerger M, Steinvorth S. Pallidal stimulation: an alternative to pallidotomy? J Neurosurg 1997; 87: 700-705.

25. Burchiel KJ. Thalamotomy for Movement Disorders. In: Gildenberg PLGE, ed. Neurosurgery Clinics of North America - Functional Neurosurgery. W.B. Saunders Company, Philadelphia, 1995: 55-72.
26. Galvez-Jimenez N, Lozano AM, Duff J, et al. Bilateral pallidotomy pronounced amelioration of incapacitating levodopa-induced dyskinesias but accompanying cognitive decline. Mov Disord 1996; 11: 242

27. Schuurman PR, De Bie RMA, Speelman JD, Bosch DA. Bilateral posteroventral pallidotomy in advanced Parkinson's disease in three patients. Mov Disord 1997; 12: 752-755.

28. Lozano A, Hutchison W, Kiss Z, et al. Methods for microelectrodeguided posteroventral pallidotomy. J Neurosurg 1996; 84: 194202.

29. Lozano AM, Lang AE, Galvez-Jimenez N, et al. Effect of GPi pallidotomy on motor function in Parkinson's disease. Lancet 1995; 346: 1383-1387.

30. Fahn S, Elton RL, Members of the UPDRS Development Committee. Unified Parkinson's Disease Rating Scale. In: Fahn S, Marsden CD, Goldstein M, Calne DB, eds: Recent Development in Parkinson's disease. Vol. 2 Florham Park, New Jersey: Macmillan, 1987: 153-163.

31. Goetz C, Stebbins GT, Shale HM, et al. Utility of an objective dyskinesia rating scale for Parkinson's disease: inter- and intrarater reliability assessment. Mov Disord 1994; 9: 390-394.

32. Alexander GE, Crutcher MD. Functional architecture of basal ganglia circuits: neural substrates of parallel processing. Trends Neurol Sci 1990; 13: 266-271.

33. Alexander GE, DeLong MR, Strick PL. Parallel organization of functionally segregated circuits linking basal ganglia and cortex. Ann Rev Neurosci 1986; 9: 357-381.

34. Alexander GE, Crutcher MD, DeLong MR. Basal ganglia-thalamocortical circuits: parallel substrates for motor, oculomotor, "prefrontal" and "limbic" functions. Prog Brain Res 1990; 85: 119-146.

35. Crossman AR. Primate models of dyskinesia: the experimental approach to the study of basal ganglia-related involuntary movement disorders. Neuroscience $1987 ; 21: 1-40$.

36. Marsden CD, Obeso JA. The functions of the basal ganglia and the paradox of stereotaxic surgery in Parkinson's disease. Brain 1994; 117: 877-897.

37. Davis KD, Taub E, Houser D, et al. Globus pallidus stimulation activates the cortical motor system during alleviation of parkinsonian symptoms. Nature Med. 1997; 3: 671-674.

38. Limousin P, Greene J, Pollak P, et al. Changes in cerebral activity pattern due to subthalamic nucleus or internal pallidum stimulation in Parkinson's disease. Ann Neurol 1997; 42: 283-291.

39. Bejjani B, Damier P, Arnulf I, et al. Pallidal stimulation for Parkinson's disease - Two targets? Neurology 1997; 49: 1564-1569.

40. Krack P, Pollak P, Limousin P, et al. Opposite motor effects of pallidal stimulation in Parkinson's disease. Ann Neurol 1998; 43: 180-192.

41. Krack P, Pollak P, Limousin P, Benazzouz A, Benabid AL. Stimulation of subthalamic nucleus alleviates tremor in Parkinson's disease. Lancet 1997; 350: 1675-1675.

42. Tröster AI, Fields JA, Wilkinson SB, et al. Unilateral pallidal stimulation for Parkinson's disease: neurobehavioral functioning before and 3 months after electrode implantation. Neurology 1997; 49: 1078-1083. 